\title{
BibGlimpse: The case for a light-weight reprint manager in distributed literature research
} Thomas Tüchler*1, Golda Velez ${ }^{2}$, Alexandra Graf ${ }^{1}$ and David P Kreil ${ }^{1}$

\author{
Address: ${ }^{1}$ Chair of Bioinformatics, Boku University, AT-1190 Muthgasse 18, Vienna, Austria and ${ }^{2}$ Internet WorkShop, 2921 South Cottonwood \\ Lane, Tucson, AZ 85713, USA \\ Email: Thomas Tüchler* - thomas.tuechler@boku.ac.at; Golda Velez - gv@btuscon.com; Alexandra Graf - alexandra.graf@boku.ac.at; \\ David P Kreil - bibglimpse08@boku.ac.at \\ * Corresponding author
}

Published: I October 2008

BMC Bioinformatics 2008, 9:406 doi:10.1186/147|-2105-9-406

This article is available from: http://www.biomedcentral.com/I47I-2/05/9/406

C) 2008 Tüchler et al; licensee BioMed Central Ltd.

This is an Open Access article distributed under the terms of the Creative Commons Attribution License (http://creativecommons.org/licenses/by/2.0), which permits unrestricted use, distribution, and reproduction in any medium, provided the original work is properly cited.
Received: I April 2008

Accepted: I October 2008

\begin{abstract}
Background: While text-mining and distributed annotation systems both aim at capturing knowledge and presenting it in a standardized form, there have been few attempts to investigate potential synergies between these two fields. For instance, distributed annotation would be very well suited for providing topic focussed, expert knowledge enriched text corpora. A key limitation for this approach is the availability of literature annotation systems that can be routinely used by groups of collaborating researchers on a day to day basis, not distracting from the main focus of their work.
\end{abstract}

Results: For this purpose, we have designed BibGlimpse. Features like drop-to-file, SVM based automated retrieval of PubMed bibliography for PDF reprints, and annotation support make BibGlimpse an efficient, light-weight reprint manager that facilitates distributed literature research for work groups. Building on an established open search engine, full-text search and structured queries are supported, while at the same time making shared collections of annotated reprints accessible to literature classification and text-mining tools.

Conclusion: BibGlimpse offers scientists a tool that enhances their own literature management. Moreover, it may be used to create content enriched, annotated text corpora for research in textmining.

\section{Background}

The published biomedical literature is growing at a tremendous pace $[1,2]$. Although access has increased considerably with the availability of most published research in electronic form (typically in the Portable Document Format, PDF), researchers now face a considerable challenge in organizing and managing comprehensive and up to date manuscript collections.

\section{Existing literature management tools}

Currently, a wide range of software is offered for searching and organizing published manuscripts. With approaches ranging from open-source bibliography managers for the desktop to professional online abstracting services, supported feature sets differ substantially (see Table 1).

While Google Scholar and other web search engines provide a full-text index of public documents online [3], there is no mechanism supporting personal collections of 
Table I: Feature comparison

\begin{tabular}{|c|c|c|c|c|c|c|c|c|c|c|c|}
\hline & $\begin{array}{l}\text { Management } \\
\text { of personal } \\
\text { collections }\end{array}$ & $\begin{array}{l}\text { Full-text } \\
\text { search }\end{array}$ & $\begin{array}{l}\text { Bibliography- } \\
\text { PDF match } \\
\text { automated }\end{array}$ & $\begin{array}{l}\text { Personal } \\
\text { annotations }\end{array}$ & $\begin{array}{l}\text { Shared } \\
\text { collections }\end{array}$ & $\begin{array}{l}\text { Approximate } \\
\text { search } \\
\text { patterns }\end{array}$ & $\begin{array}{l}\text { Search with } \\
\text { synonyms }\end{array}$ & $\begin{array}{l}\text { Index } \\
\text { browsing }\end{array}$ & $\begin{array}{l}\text { Ease of } 1 / O \\
\text { with external } \\
\text { tools } 10\end{array}$ & $\begin{array}{l}\text { Free use or } \\
\text { open-source }\end{array}$ & Requirements \\
\hline BibGlimpse & Yes & Yes & Yes & Yes & Yes & Yes & No & No & Yes/Yes & Yes'I & $\begin{array}{l}\text { Bash, Perl, } \\
\text { Apache }\end{array}$ \\
\hline \multicolumn{12}{|l|}{$\begin{array}{l}\text { Abstracting } \\
\text { services }\end{array}$} \\
\hline PubMed & $\operatorname{Prt}^{1}$ & No & $\operatorname{Prt}^{3}$ & No & No & No & Prt & Yes & No/Yes & Yes & Web \\
\hline ISI Web of & $\operatorname{Prt}^{1}$ & No & No & No & No & No & No & Yes & No/Yes & No & Web \\
\hline \multicolumn{12}{|l|}{$\begin{array}{l}\text { Science } \\
\text { Search engine }\end{array}$} \\
\hline $\begin{array}{l}\text { Google } \\
\text { Scholar }\end{array}$ & No & Yes $^{2}$ & $\operatorname{Prt}^{3}$ & No & No & No & No & No & No/No & Yes & Web \\
\hline \multicolumn{12}{|l|}{$\begin{array}{l}\text { Reference } \\
\text { managers }\end{array}$} \\
\hline EndNote & Yes & No & $\mathrm{No}^{4}$ & Yes & No & No & No & Prt & No/Yes & No & Win/Mac \\
\hline RefBase & Yes & No & No & Yes & Yes & No & No & Yes $^{9}$ & No/Yes & Yes & XAMPPI2 \\
\hline iPapers & Yes & Yes & $\mathrm{No}^{5}$ & Yes & No & No & No & No & No/Yes & Yes & Mac \\
\hline \multicolumn{12}{|l|}{$\begin{array}{l}\text { Social } \\
\text { bookmarking }\end{array}$} \\
\hline CiteULike & Yes & No & $\mathrm{No}^{6}$ & $\mathrm{Prt}^{7}$ & Yes & No & No & Tags & $\mathrm{No} / \mathrm{Prt}$ & Yes & Web \\
\hline Connotea & Yes & No & $\mathrm{No}^{6}$ & Yes & Yes & No & No & Tags & No/Prt & Yes & Web \\
\hline \multicolumn{12}{|l|}{ Digital library } \\
\hline Greenstone & Yes & Yes & No & $\mathrm{Yes}^{8}$ & Yes $^{8}$ & Yes & No & Yes & Yes/Yes & Yes & Perl, Apache \\
\hline
\end{tabular}

Feature comparison between BibGlimpse and other bibliographic software tools. 'Prt' indicates that a feature is partly supported. ' Searches and selected references can be stored in collections. ${ }^{2}$ Covers the first $120 \mathrm{kB}$ of open access papers and a non-disclosed list of publishers; all recent articles by Elsevier publications are, e.g., excluded. PubMed is indexed with a lagtime of up to a year. ${ }^{3}$ Linking bibliography to full text; not the other way around. ${ }^{4}$ For a given reference an automated online search for the corresponding full-text can be performed. ${ }^{5}$ Requires file named PMID.pdf, where PMID is the PubMed ID, to download bibliography from PubMed. ${ }^{6}$ Needs link to website, not link to PDF. Retrieval is not generic, but publisher site tailored. ${ }^{7}$ Notes are not searchable. ${ }^{8}$ Greenstone is a tool to build digital libraries, so library needs to be designed first. 9 MySOL database can be queried directly by passing MySOL search strings. 10 Input means that results of external tools can easily be input into the system for subsequent integrated analysis and searches. Output means that data in the system can be output to external tools. ${ }^{11}$ Code free for non-profit and academic use. ${ }^{12}$ Package providing PHP, MySQL and Apache for different platforms. 
online documents to be annotated or searched, and many articles are not even publicly available. Desktop search tools, in turn, do not facilitate the sharing of documents and their annotation between collaborators. Dedicated bibliography management software like RefBase and WikIndx (see [4]) or the popular commercial tools EndNote [5] and RefWorks [6] have a different focus: there is no support for full-text search and, depending on the tool, complex queries are not supported or data cannot be shared online. On the other hand, full-featured software for digital libraries (like Greenstone [7]) is not only difficult to set up but also impractical for casual use. Long forms to fill in, which make filing a PDF reprint considerably more tedious than just saving it to disk, reduce acceptance in day-to-day work.

With these challenges and unmet needs in mind, we here introduce the concept of a light-weight reprint manager for the joint creation and exploitation of content enriched collections of expert annotated full-text reprints. The BibGlimpse implementation provides a simple framework for distributed literature research especially designed for this purpose. In particular, besides allowing full-text searches on manuscript collections, the support for searchable personal annotations and the ability of sharing these with colleagues are key features of the system. The automated creation of bibliographic records for a simple PDF reprint, moreover, substantially facilitates the uptake of the system. Such automatic retrieval of bibliographic records from full text PDF files is a feature that has, to the best of our knowledge, not yet been implemented elsewhere.

\section{Implementation}

A defining design requirement for BibGlimpse was that users can file a new PDF reprint by simply saving or copying it to disk, or by uploading it online without being prompted to fill in any forms. The lightweight filesystembased approach also means that users can easily import an entire collection of reprints using just a single command or 'drag and drop' operation for copying the directories of their PDF files. Users can of course also manually edit or add bibliographic records, personal annotation, and supplement files. Medline, BibTeX, and RIS formats are supported. When a Medline or RIS format record is available but not a BibTeX record, the system automatically creates one. Extending standard Webglimpse functionality ( $c f$. Figure 1), the index is updated in the background to cover the bibliographic record, any user annotation, and the extracted full-text of an article.

\section{Automated Medline retrieval}

When a new reprint is detected in the indexed file system, BibGlimpse extracts the plain text from the PDF file and constructs queries to automatically obtain a matching bibliographical record from PubMed. Queries are compiled with a generic pattern recognition approach, avoiding a need to prepare numerous journal specific templates for the extraction of bibliographic information from the PDF [8]. To this end, BibGlimpse first discards unspecific or irrelevant text sections, i.e. lines that are presumably not suited for constructing a meaningful PubMed query. This heuristic filter comprises simple rules, like a line must at least contain five characters, two words and at least one word with more than four letters. It also excludes lines that are likely to contain figure captions ('Fig.'), contact or company addresses ('Inc.'). Moreover, since citations can easily confound query construction (and are often found on the first page of articles where articles do not start on a fresh page), lines matching regular expressions that target such citations are equally removed.

By means of further heuristics, several features are then extracted from the prefiltered text in order to come up with query strings for the putative title, authors and abstract of the reprint in question: While identification of the putative manuscript title mainly focuses on the position in the text (e.g., the title is assumed to be located within the top lines of the document, it is supposed not to exceed a certain length and to be separated from the remainder by blank lines), features relevant for finding the manuscript's authors strongly rely on punctuation. To illustrate that, consider the following example line, obtained after converting a reprint PDF to text:

Xiaolei Yu,1 Milorad Susa,2 Cornelius Knabbe,2 Rolf D. Schmid, 1 and Till T. Bachmann 1*

Without knowing that 'Xiaolei' or 'Cornelius' are names, we can characterize this line by the following features: it contains 6 delimiters (including punctuation like the comma and ampersand symbols as well as the word 'and'), 5 author affiliation symbols including 1 corresponding author asterisk, 2 middle name initials (e.g. one in Rolf D. Schmid), and 10 out of 13 words start with capital letters (not counting the initials). Based on such observations, we extract the following 8 characteristic features: per-word ratios for delimiters $(6 / 13$ in this example), footnote symbols (5/13), middle name initials (2/ $13)$, and words starting with capital characters (10/13); moreover, indicators for the existence of an asterisk (1), colon characters (0), and whether the whole line is written in capital letters $(0)$, as well as the distance to the next putative headline (counted in lines).

To construct a PubMed query string with the putative authors of a manuscript, these features are first computed for each line in the PDF and then exploited to identify 'author lines', i.e. lines containing the authors of a manuscript. For classification, we trained a radial basis function 


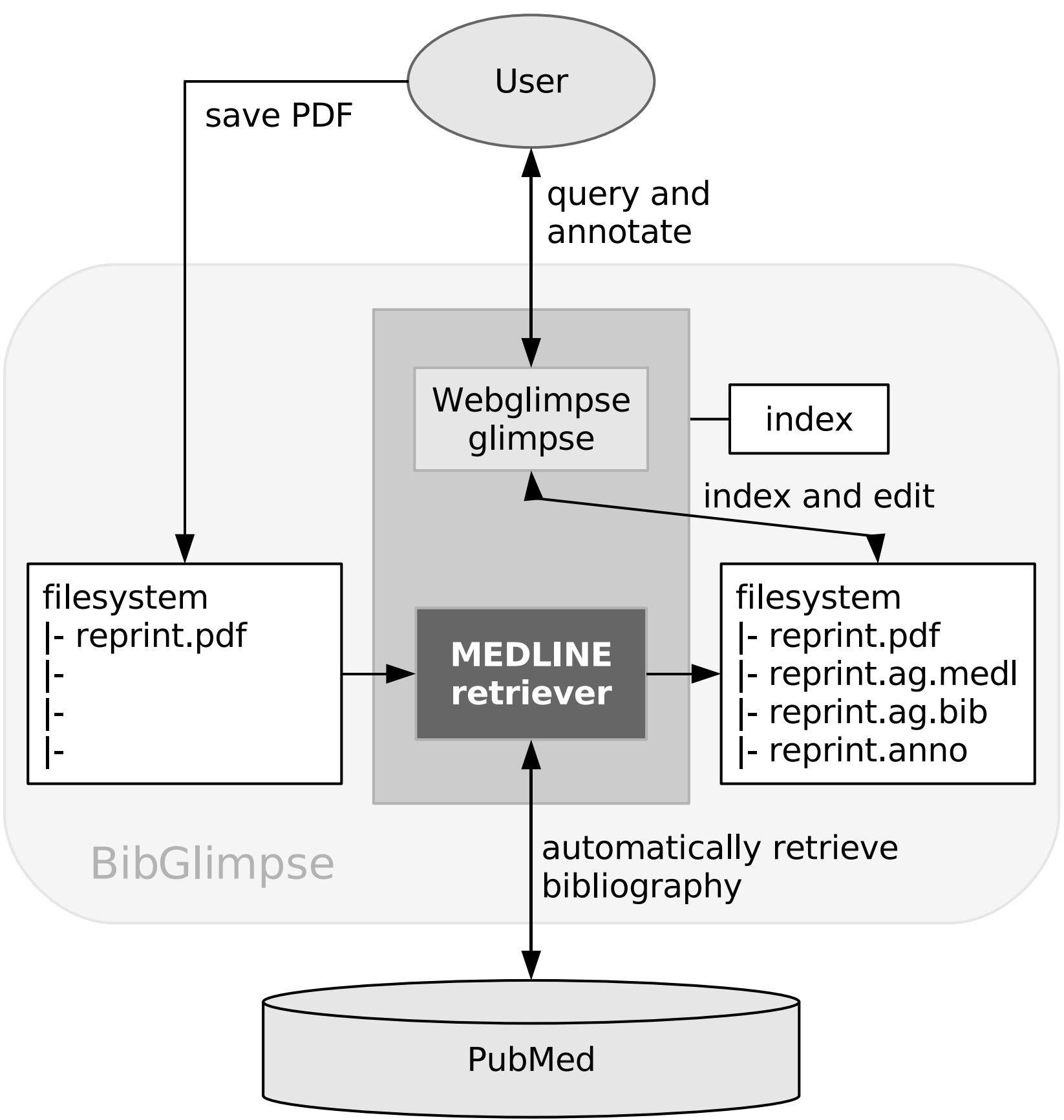

\section{Figure I}

BibGlimpse scheme. The figure schematically illustrates how BibGlimpse incorporates automated Medline retrieval into the Webglimpse search environment. Saved PDFs are automatically matched with a Medline record and indexed. For integration with external tools, all data are directly available in flatfile format.

kernel support vector machine (SVM) on different representative training sets of 20 author and 20 other text lines. Using only the above 8 features, the finally selected support vectors achieved a respectable recall of $95 \%$ true pos- itives (TP) with only $8 \%$ false positives (FP) as assessed in an independent test set of over 2000 candidate lines. A robust typical recall of more than $85 \%$ TP with about $10 \%$ $\mathrm{FP}$ in an investigation of alternative random training data 
indicated a well chosen feature set. Details regarding the classifier and the test corpus employed can be found in the online Supplement.

The good performance achieved in author-line identification allows targeted PubMed queries for authors with only a reasonable number of FP non-author text queries submitted. At this point, we wish to emphasize that PubMed hits are not only gathered by querying for the putative authors, but also from searching for the presumed title, abstract, and the digital object identifier, if available. The aim of the described filters is therefore not to extract the true bibliography directly from the manuscript, but rather to construct a set of query strings, that allow a retrieval of this information from PubMed with as few requests as possible.

Eventually, even obtaining a unique PubMed hit for a query string is not sufficient to assure that manuscript and bibliography match. Each retrieved Medline record is thus additionally cross-checked against the extracted full-text by reverse queries of title, authors and abstract.

Technically, the retrieval of Medline records from PubMed was implemented in Perl, such that it can be run from a single stand-alone script. This makes the feature easily accessible for other environments.

\section{Results and discussion Performance}

The real-world performance of the complete system for the automated retrieval of bibliographic records was assessed on a test set of over 1000 PubMed listed manuscripts covering about 200 different journals. BibGlimpse was able to retrieve the correct PubMed records for $95 \%$ of these manuscripts with only $0.5 \%$ spurious hits and the remaining $4.5 \%$ being tagged as not-found. This shows that the combination of multiple heuristic queries and the cross-checking process yields an overall robust performance. There are some cases, however, where retrieval might not succeed. Consider the following text line returned from a 'pdftotext' conversion:

Gene Expression Profiles in Formalin-Fixed, ParaffinEmbedded Tissues Obtained with a Novel Assay for Microarray Analysis, Marina Bibikova,1 Joanne M. Yeakley,1 Eugene Chudin,1 Jing Chen,1 Eliza Wickham,1 Jessica WangRodriguez, 2 and Jian-Bin g Fan1* (1 Illumina, Inc., San Diego, CA)

In this example, no newline character separates the title of the manuscript from the authors and their addresses. Moreover, the corresponding Medline entry (PMID 15563488) lists 'paraffin-embedded tissues' in the title, instead of the poorly converted 'ParaffinEmbedded' in the text version of the PDF. The cross-checking step may in such cases find no match. Also, single PDF files containing multiple short comments or letters to the editor may be assigned to the bibliography for the first comment or letter. Finally, the system may not be able to distinguish preprints from actually published manuscripts if they have the same title, authors, and abstract. In summary, however, these limitations only apply to a very small fraction of reprints files.

\section{Application}

Freeing researchers from a need to look up or enter bibliographical records and giving them an opportunity of annotating their reprints together with full-text query capabilities not only raises acceptance of the system in day-to-day usage but has profound practical implications for knowledge discovery, sharing, and retrieval. We illustrate this with a few examples (Figure 2). Important information, such as the cell line types employed, is often not contained in the abstract but can be queried by full-text search (e.g., a query for cell line HCT116, Figure 2). Other information may even only be implicit in the full manuscript text but can be captured explicitly by user annotation (e.g., user annotation as 'p53 wildtype').

The challenge of searching natural language text, of course, remains. Depending on the application domain, it may be valuable to consider extending the free-form annotation of articles by keywords from ontologies with controlled vocabularies or controlled subsets of natural language [9]. External tools can easily be integrated to either automatically generate these, or at least assist the user in a manual curation process [10]. This is much facilitated by having all internally stored information available as plain text files on disk. Through structured query support such additional fields can be queried directly. Having straightforward means for the integration of textmining tools thus allows future developments to further assist users in extracting searchable knowledge from their annotated reprint collections.

The availability of installation support and minimal software prerequisites make BibGlimpse accessible for small groups of collaborating researchers. Building on Webglimpse [11], our system inherits a search engine that has been actively maintained and supported for over ten years, is easy to install and maintain using an administrative web interface, and only needs Perl, a running web server and a utility to extract plain text from PDF files, e.g., from 'xpdf' [12].

\section{Implications for text-mining}

Application of BibGlimpse can make expert annotated literature collections available to text-mining. On the one hand, richly annotated text corpora are valued for training 

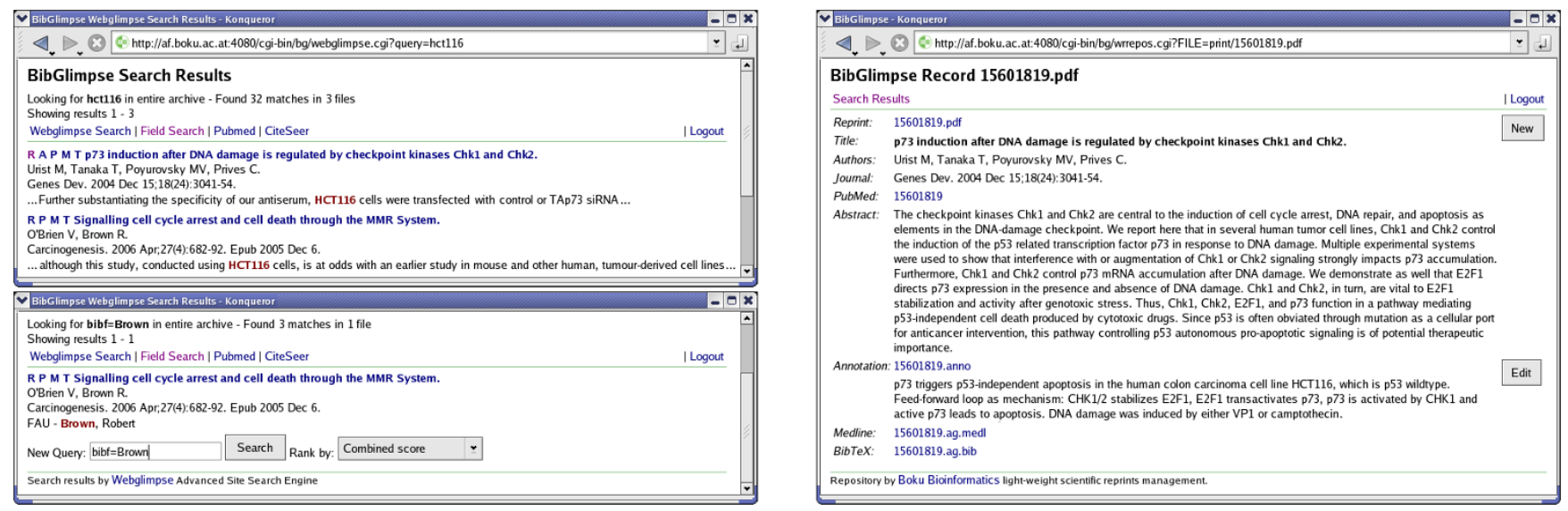

Figure 2

BibGlimpse impressions. The upper left panel shows results of a full-text query for 'HCTI I6'. A corresponding repository record is depicted on the right, where a domain expert captured relevant information in free-form annotation. Note that the short URLs can easily be sent to collaborating researchers. The lower-left panel demonstrates a structured query, searching only the bibliographic records for 'Brown', which avoids picking up this frequent term in the full-text, e.g., from the citations section.

and testing algorithms [13-15]. On the other hand, textmining applications benefit even from coarse auxiliary information [16-18]. Yet, the ability to devote scarce resources to annotating literature is often the limiting factor constraining especially machine learning approaches $[19,20]$.

Also it is recognized that full manuscript texts provide more information than abstracts [21-24] and that information retrieval is more successful in domain specific collections [18]. Obtaining access to a comprehensive range of full-text articles, however, can be troublesome due to copyright issues, and the identification of relevant journals can be quite difficult in an interdisciplinary field [25]. Specialist researchers are actually best placed for compiling representative domain specific collections of content enriched full-text articles.

Facilitating the creation process of such annotated collections could hence significantly advance biomedical textmining. So far, most researchers collect, freely [26] or by subscription, manuscripts of interest to their research area from multiple journals, typically by storing the PDF reprint on their computers. They maintain their personal collections of reprints, notes, and bibliographic records using a variety of tools, ranging from simple text editors or spreadsheets to commercial bibliography management software. But while extensively covering a particular area of interest, representing valuable resources of domain knowledge, such personal repositories are currently hard to search or exploit.
BibGlimpse offers researchers a tool enhancing their own routine literature management and supports the creation of shared domain-specific collections of annotated fulltext manuscripts. This benefits both biological researchers as well as the text-mining community.

\section{Conclusion}

Considering the benefits of the system's automation and query capabilities in supporting shared literature research, together with its straightforward interfacability with literature classification and text-mining tools, we have reason to expect that BibGlimpse will be widely adopted. We are confident that the concept of a light-weight reprint manager demonstrated in BibGlimpse will transform how research groups collect, manage, and share knowledge from literature research.

\section{Availability and requirements \\ Project name: BibGlimpse}

Project home page: http://bioinf.boku.ac.at/bibglimpse

Operating system(s): UNIX

Programming language: Perl, Bash

Other requirements: Apache 2.2.6 or higher, Perl 5.8.6 or higher, pdftotext (e.g., from xpdf 3.01 or higher)

License: $\quad$ http://webglimpse.net/sublicensing/licens 
Any restrictions to use by non-academics: licence needed

\section{Authors' contributions}

TT implemented the automated PubMed entry retrieval and drafted the manuscript, GV helped with integrating this feature into the Webglimpse search engine, AG contributed the BibGlimpse on Cygwin package, while DPK devised the concept of BibGlimpse, participated in its implementation and helped to draft the manuscript. All authors read and approved the final manuscript.

\section{Acknowledgements}

The Boku Bioinformatics group acknowledges support by the Vienna Science and Technology Fund (WWTF), the Austrian Centre of Biopharmaceutical Technology (ACBT), Austrian Research Centres Seibersdorf (ARCS), and Baxter AG. TT acknowledges support by the GEN-AU project Bioinformatics Integration Network of the Austrian Federal Ministry of Science and Research program.

\section{References}

I. Ananiadou S, Kell DB, Tsujii Jl: Text mining and its potential applications in systems biology. Trends Biotechnol 2006, 24( I 2):57|-579.

2. Jensen LJ, Saric J, Bork P: Literature mining for the biologist: from information retrieval to biological discovery. Nat Rev Genet 2006, 7(2): I19-29.

3. Giustini D, Barsky E: A look at Google Scholar, PubMed and Scirus: comparisons and recommendations. JCHLAIJABSC 2005:85-89.

4. sourceforge [http://sourceforge.net]

5. EndNote [http://www.endnote.com]

6. RefWorks [http://www.refworks.com]

7. Greenstone [http://greenstone.org]

8. Müller HM, Kenny EE, Sternberg PW: Textpresso: an ontologybased information retrieval and extraction system for biological literature. PLOS Biol 2004, 2(I I):e309.

9. Kuhn T, Royer L, Fuchs N, Schroeder M: Improving text mining with controlled natural language: A case study for protein interactions. DILS LNBI 2006.

10. Rebholz-Schuhmann $D$, Kirsch H, Couto F: Facts from text-is text mining ready to deliver? PLoS Biol 2005, 3(2):e65.

II. Velez G: The Searchable Site. Linux Gazette 2006, I47:.

12. xpdf [http://www.foolabs.com/xpdf/]

13. Kim JD, Ohta T, Tsujii J: Corpus annotation for mining biological events from literature. BMC Bioinformatics 2008, 9:10.

14. Kim JD, Tsujii J: Corpora and their Annotation. In Text Mining for Biology and Biomedicine Edited by: Ananiadou S, McNaught J. Artech House; 2006: 179-2II.

15. Cohen K: Corpus design for biomedical natural language processing. Proceedings of the $A C L$ workshop on Linking Biological Literature, Ontologies and Databases: mining biological semantics, Association for Computational Linguistics 2005:38-45.

16. Bockhorst J, Craven M: Exploiting Relations Among Concepts to Acquire Weakly Labeled Training Data. In Proceedings of the 19th International Conference on Machine Learning Morgan Kaufman; 2002:43-50.

17. Suomela BP, Andrade MA: Ranking the whole MEDLINE database according to a large training set using text indexing. BMC Bioinformatics 2005, 6:75.

18. Lee $M$, Wang W, Yu H: Exploring supervised and unsupervised methods to detect topics in biomedical text. BMC Bioinformatics 2006, 7:140.

19. Hunter L, Cohen KB: Biomedical language processing: what's beyond PubMed? Mol Cell 2006, 2 I (5):589-94

20. Wilbur WJ, Rzhetsky A, Shatkay H: New directions in biomedical text annotation: definitions, guidelines and corpus construction. BMC Bioinformatics 2006, 7:356

21. Corney DP, Buxton BF, Langdon WB, Jones DT: BioRAT: extracting biological information from full-length papers. Bioinformatics 2004, 20(17):3206-13.
22. Ray S, Craven M: Learning statistical models for annotating proteins with function information using biomedical text. BMC Bioinformatics 2005, 6(SuppI I):SI8.

23. Saric J, Jensen L, Ouzounova R, Rojas I, Bork P: Extraction of regulatory gene/protein networks from Medline. Bioinformatics 2006, 22(6):645-50.

24. Natarajan J, Berrar D, Dubitzky W, Hack C, Zhang Y, DeSesa C, Van Brocklyn JR, Bremer EG: Text mining of full-text journal articles combined with gene expression analysis reveals a relationship between sphingosine-I-phosphate and invasiveness of a glioblastoma cell line. BMC Bioinformatics 2006, 7:373.

25. Postma E: Inflated impact factors? The true impact of evolutionary papers in non-evolutionary journals. PLOS ONE 2007, 2(10):e999.

26. Wren JD: Open access and openly accessible: a study of scientific publications shared via the internet. BMJ 2005, 330(7500): I I 28

Publish with Bio Med Central and every scientist can read your work free of charge

"BioMed Central will be the most significant development for disseminating the results of biomedical research in our lifetime. "

Sir Paul Nurse, Cancer Research UK

Your research papers will be:

- available free of charge to the entire biomedical community

- peer reviewed and published immediately upon acceptance

- cited in PubMed and archived on PubMed Central

- yours - you keep the copyright
BioMedcentral 